\title{
Théologiques
}

Théologiques

\section{Garder le silence}

\section{Jean-Claude Petit}

Volume 7, numéro 2, automne 1999

Silence !

URI : https://id.erudit.org/iderudit/005021ar

DOI : https://doi.org/10.7202/005021ar

Aller au sommaire du numéro

Éditeur(s)

Faculté de théologie de l'Université de Montréal

ISSN

1188-7109 (imprimé)

1492-1413 (numérique)

Découvrir la revue

Citer ce document

Petit, J.-C. (1999). Garder le silence. Théologiques, 7(2), 3-10.

https://doi.org/10.7202/005021ar d'utilisation que vous pouvez consulter en ligne.

https://apropos.erudit.org/fr/usagers/politique-dutilisation/ 
LIMINAIRE

\section{Garder le silence}

Jean-Claude PETIT

Faculté de théologie

Université de M ontréal

Les plus grands événements - cene sont pas nos heures les plus bruyantes, mais nos heures les plus silencieuses. Ce n'est pas autour des inventeurs de fracas nouveaux, c'est autour des inventeurs de valeurs nouvelles que gravite le monde : il gravite inaudiblement.

(F. N ietzsche ${ }^{1}$ )

Lorsque nous voulons bien marquer l'importance d'un événement nous disons souvent que telle ou telle chose « a fait grand bruit », ou encore qu'elle a eu « beaucoup d'écho ». II n'est pas rare de lire à la une du journal quotidien qu'une nouvellea éclaté « comme une bombe ». Ce qui ne fait pas de bruit ne mérite pas qu'on s'y arrête. Ce qui en fait un peu peut attiser l'inquiétude : la rumeur agace. $M$ ais tant quel'affairen'a pas « éclaté » au grand jour ou qu'ellen'a pas été « ébruitée », on peut se permettre de l'ignorer, ou s'appliquer à l'« étouffer ».

Le mot de $\mathrm{N}$ ietzsche ne semble bien n'avoir d'autre intérêt que celui d'une belle fiction poétique, parole « inactuelle » sans authentique vérité. Parole étonnante, pourtant, dans laquelle une oreille un peu attentive reçoit l'écho d'une parole plus ancienne où se reconnaissent les traces, là aussi, d'une expérience originelle. II s'agit de I'antienne des vêpres du dimanche dans l'octave de la $\mathrm{N}$ ativité :

1. «Des savants». Ainsi parlait Zarathoustra. [N ietzsche Werke. Kritische Gesamtausgabe, VI, 1, p. 165] (trad. M. Betz). Cf. J.-L. CHRÉTIEN, L'arche de la parole, Paris, P.U.F., 1998, p. 62. 
«Tandis que toutes choses gardaient un profond silence, et que la nuit atteignait, dans sa course, le milieu de sa route, ta Parole toute-puissante, Seigneur, s'élança de son trône royal ». "D um medium silentium tenerent omnia... ». J ean-Louis Chrétien commente : "La plus silencieuse de toutes les heures est celle de la $\mathrm{N}$ ativité ${ }^{2}{ }^{2}$

Profond silence des commencements et des grands événements. En une étonnante complicité, la parole de la liturgie et la parole de $\mathrm{N}$ ietzsche s'inscrivent en faux contre l'illusion qui illustre si bien le désarroi de ceux qui habitent « le pays de la civilisation ». ${ }^{3}$ Le bruit est tellement devenu la mesure de ce qui compte à nos yeux que le «profond silence» est devenu pour nous, comme nous le disons si spontanément, "un silence de mort ». Alors qu'il était, pour $\mathrm{N}$ ietzsche et pour l'orant de la liturgie, l'espace même de la vie.

On dira que le silence exerce tout de même une certaine fascination et qu'il révèle ainsi son importance. $O \mathrm{n}$ en voudra pour preuve l'attrait, voire la fascination précisément, qu'exerce sur un grand nombre de contemporains, le cloître ou le désert. Les voies du silence, en effet, conduisent facilement aux berges du religieux. C'est d'ailleurs dans I'abondante littérature issue de la tradition religieuse multiforme de l'humanité que l'évocation du silence est la plus persistante et la plus riche. Le silence est par excellence l'affaire des moines. $N$ e sont-ils pas ceux qui sont « mûris à l'école du silence », commele note fort à propos Jean Leclercq ${ }^{4}$ ? A ussi, ne faut-il pas trop se surprendre si, dans I'imaginaire des contemporains, l'évocation du silence continue de parler d'ascèse et de religion, d'expérience spirituelle ou de mystique. R éalité tout aussi fascinante qu'inquiétante, parce que réservée, semble-t-il, à quelques individus privilégiés ou à quelques moments imprévisibles d'une existence qui ne sait trop quoi en faire.

J'observe en tout cas que, le plus souvent, le silence nous embête et nous gêne. Le professeur ou le conférencier ne peut pas rester quelques instants sans parler sans que l'auditoire n'exprime sa gêne et ne

2. J.-L. CHRÉTIEN, L'arche de la parole, p. 60.

3. Cf. F. NIETZSCHE, «Le pays de la civilisation». [Vom Lande der Bildung: Also Sprach Zarathustra, II. N ietzsche Werke, Kritische Gesamtausgabe VI, 4, p. 149-151].

4. J. LECLERCQ, L'amour des lettres et le désir de Dieu. Initiation aux auteurs monastiques du M oyen-Âge, Paris, Cerf, 1990 (1957), p. 146. 
s'inquiète d'une possible défaillance. Le lecteur du bulletin de nouvelles ne peut se permettre aucun silence. Les silences calculés du prédicateur faisaient rapidement grincer les bancs et tousser les fidèles. Le silence qui règne là où plusieurs sont rassemblés est rapidement suspect. $O \mathrm{n}$ ne sait pas trop comment s'introduire dans un lieu où règnele silence alors qu'on se sent d'emblée plus à l'aise s'il règne une certaine animation.

Le silence a manifestement quelque chose à voir avec la compréhension très concrète que nous avons de nous-mêmes dans nos rapports aux autres et à notre environnement. Certes, s'il y a beaucoup de bruits familiers, il y a aussi des bruits terribles et inquiétants, qui nous saisissent d'effroi et nous propulsent un moment à des limites jusqu'alors ignorées de nous-mêmes. $M$ ais l'expérience du bruit, si terrible soit-elle, engage toujours avec ellel'expérience d'un « autre », alors que dans le silence... Qu'y a-t-il dans le silence?

Si le silence exerce sur nous toujours une certaine fascination, il ne semble pas qu'il constitue une priorité pour la réflexion. Le thème, encore une fois, semble familier. On ne s'étonne pas qu'il vagabonde dans les livres de méditation et de soi-disant spiritualité, et c'est dans ces rayonnages hétéroclites qu'on acceptera de l'y chercher. À qui viendra-t-il à l'idée qu'il pourrait aussi faire l'objet d'un chapitre de I'analyse de la modernité? Q ui voudra le chercher dans la réflexion critique sur notre expérience de « postmodernes »? De toute évidence, I'actualité impose d'autres tâches à la pensée et il serait irresponsable de ne pas s'y engager. La théologie ne fait pas ici exception. La destruction de l'environnement, la force destructrice de la " mondialisation », le nivellement des cultures, l'impérialisme de l'économique, la violence du politique, la manipulation del'éducation et de la culture par les intérêts du capital, la culture de l'éphémère, la dictature des certitudes... Faut-il vraiment consacrer un numéro de revue dite " savante » au thème du silence alors que nous sommes toujours à pied d'oeuvre à l'égard de toutes ces urgences?

Et pourtant! Par delà toutes les considérations évoquées plus haut, au point de départ : un constat, brutal, formulé avec force et beaucoup de lucidité par George Steiner dans son dernier livre : « Le bruit - industriel, technologique, électronique, amplifié à hauteur de la folie ( « rave ») - est la peste bubonique du populisme capitaliste. Pas seulement dans I'O ccident saturé de médias, mais jusque dans les baraques de tôle ondulée des bidonvilles africains ou parmi les multitudes de 
Shanghai. Seuls parviennent à s'entendre les privilégiés ou les sourds $\gg .5$ Le drame réside peut-être dans le fait que ceux qui en sont le plus touchés ne paraissent pas s'en rendre compte. Contre le bruit insupportable, on s'isole derrière les écouteurs de son « baladeur », jusque dans les salles de travail des bibliothèques universitaires. Et ce sont désormais les aiguilles d'instruments de mesure, et non pas les oreilles des citoyens, qui décident des seuils de tolérance au-delà desquels les administrations accepteront d'engager des fonds pour construire, par exemple, des murs antibruit. Le bruit est devenu l'agression la plus omniprésente et la plus avilissante que la civilisation technique ait produite. $\mathrm{N}$ ulle part son caractère destructeur n'apparaît plus clairement que dans son alliance avec le militaire.

$M$ ais il y a aussi autre chose, qui ne relève pas cette fois de l'expérience pour ainsi dire immédiate. II s'agit plutôt d'une « hypothèse », ou d'une intuition, mais combien de fois vérifiée : à force d'identifier le silence au « religieux », dans la mesure où celui-ci en est venu à être compris de plus en plus comme un arrière-monde sans signification effective pour le seul véritable monde de l'existence publique quotidienne, nous sommes devenus incapables de reconnaître dans le silence une posture humaine essentielle. N ous avons fait du silence une réalité extraordinaire, réservée en quelque sorte à quelques initiés, en des lieux exclusifs, à la marge sinon carrément en dehors du quotidien. 0 u peutêtre est-ce la force disséminatrice du bruit lui-même qui nous a peu à peu fait perdre de vue que, si le silence est le mode d'advenir du divin en même temps que le chemin qui y conduit - comme le pensaient Augustin et $M$ aître Eckhart - c'est qu'il est aussi le chemin qui nous conduit à nous-mêmes et le lieu où il nous est possible d'advenir.

Sans doute une réelle conversion est-elle nécessaire pour retrouver ainsi la place du silence dans le quotidien de la vie. Peut-être même que cette redécouverte n'est plus vraiment en notre pouvoir, mais ne peuton pas s'exercer à la préparer et, s'y exerçant, en faire déjà peut-être un peu l'expérience? Je crois qu'il y a urgence à réapprendre à écouter le silence au plus près de notre expérience quotidienne de nous-mêmes et de notre monde. $\mathrm{O}$ u tout au moins à en laisser monter le désir en nous. C'est pourquoi on ne proposera pas en entrée une réflexion sur l'appartenance du silence et de la prière, du silence et du divin, mais plutôt ce que David Le Breton appelle simplement une " anthropologie du

5. G. Stein ER, Errata. Récit d'une pensée, Paris, Gallimard, 1998, p. 198. 
silence ». En 1997, il avait fait paraître un essai remarquable sur le silence. ${ }^{6}$ " Le silence, y notait-il, n'est pas seulement une certaine modalité du son, il est d'abord une certaine modalité du sens » (p. 144). Or tout l'enjeu devient visible dans ce constat déjà évoqué : « La modernité est l'avènement du bruit » (p. 14). David Le Breton reprend et poursuit ici les réflexions de ce livre important.

Le silence est une posture humaine. J ean-L ouis Chrétien observe cependant à ce propos quelque chose de fondamental, qui n'apparaît pas assez souvent dans les réflexions sur le silence alors qu'elle en découvre pourtant toute la portée humaine. Chrétien cite Samuel Beckett : «Et en vérité ce n'est pas tout de garder le silence, mais il faut voir aussi le genre de silence qu'on garde ». ${ }^{7}$ " II n'est pas rigoureux, continue Chrétien, d'étudier les formes du silence comme si nous n'étions nous-mêmes nulle part, et le rapport de l'homme au silence et aux silences, ainsi que les actes par lesquel s ce rapport s'établit, donnent le seul principe possible d'une typologie ».8

Le silence peut être la forme que prend le pardon mais il peut être aussi le premier pas du meurtrier. «Le silence du bourreau » est le titre de la contribution de Serge $C$ antin. En nous invitant à réfléchir sur « le crime contre l'humanitéau $X X^{e}$ siècle », Cantin veut nous rappeler certes, que « quelque chose qui vient vous tuer commence toujours par se taire » (Christian Bobin), ce qui devrait lester d'un peu d'inquiétude toute réflexion trop empressée à fuir les bruits du monde pour chercher refuge dans le silence des dieux. $M$ ais son analyse pourrait aussi nous aider à prendre conscience que s'il existe bel et bien « un silence qui fait signe vers une effroyable dépersonnalisation de la conscience », celui-ci n'est pas qu'une subtile tentation à laquelle, avec un peu d'effort, il nous serait toujours possible d'échapper, mais une blessure, une rupture permanente qui ne nous permet plus, après Auschwitz, de croire, ni en Dieu ni en l'homme, de la même manière qu'avant.

En 1965, à l'occasion de l'ouverture de l'exposition de l'Union des artistes de la région $\mathrm{R}$ hein- $\mathrm{N}$ eckar à $\mathrm{H}$ eidelberg, $\mathrm{H}$ ans-G eorg $\mathrm{G}$ adamer prononça une conférence sur un thème à première vue fort étonnant et

6. D. Le Breton, D u silence. Essai, Paris, Éditions M étailié, 1997, 283 p.

7. S. BECKETT, L'innommable, Paris, C. Bourgeois, 1971, p. 37, dans J.-L.

CHRÉTIEN, L'arche de la parole, p. 63.

8. Ibid., p. 64. 
qui n'avait été jusque là que rarement abordé. Son intervention porte le titre « du silence des tableaux ». Trois ans plus tôt était paru un ouvrage d'A rnold G ehlen intitulé « zeitbilder », « images du temps », ou mieux peut-être, « images de l'époque». Gadamer en avait fait une recension fort substantielle dans la 'Philosophische R undschau' dans laquelle il avait d'entrée de jeu signalé comment Gehlen parlait du " silence marqué d'esprit », " geisterhafte Stummheit », qui avait enveloppé la peinture depuis le post-impressionnisme. ${ }^{9} \mathrm{G}$ adamer reprend donc en 1965 cette analyse qu'il introduit alors ainsi :

J'aimerais proposer comme « eidos », le point de vue à partir duquel la production picturale contemporaine se représente et s'interprète ellemême. J'aimerais parler du silence qui advient dans le langage pictural, de la façon dont les tableaux se taisent. Se taire ne veut pas dire ne rien avoir à dire. Se taire (« verstummen ») est au contraire une manière de parler. Le mot allemand pour «muet » («stumm») est proche d'un autre mot allemand, de «balbutier» (« stammeln ») : l'émouvante pénurie du balbutiement ne consiste pas dans les faits que la personne qui balbutie n'ait rien à dire, mais plutôt en ce qu'elle ait beaucoup, voire trop à dire à la fois. Elle ne trouve pas les mots qu'il faut en raison de l'urgence et de l'abondance de ce qu'elle aurait à dire. Et lorsque nous disons que quelqu'un se tait, nous ne voulons pas seulement dire qu'il s'arrête de parler. Le fait de se taire nous rapproche de ce qui est à dire comme de ce qui nous incite à chercher des mots nouveaux. Si l'on se rappelle la riche éloquence et les coloris somptueux des peintures classiques suspendues aux murs de nos musées, qui parlent à voix forte et expressive, et qu'on a devant soi des tableaux contemporains, on a alors effectivement l'impression que ceux-ci se taisent. La question s'impose de savoir ce qui a conduit les tableaux modernes à se taire et à nous assaillir par là même de leur propre éloquence, silencieuse. ${ }^{10}$

9. Recension de H.-G. GADAM ER : «Begriffene M alerei? Zu A. Gehlen : Zeit-Bilder »Philosophische R undschau 10 (1962) 21-30. Repris dans Interpretationen. (K leine Schriften, II), Tübingen, J.C.B. M ohr, 1979, p. 218226, puis dans le t. 8 des « G esammelte Werke » (Tübingen, J.C.B. M ohr, 1993), p. 305-314.

10. «Du silence des tableaux », dans H.-G. GADAM ER, L'actualité du beau. (Trad. Elfie Poulain). A ix-en-Provence, Alinea, 1992, p. 154-155. (Texte allemand: "Vom Verstummen des Bildes», dans Interpretationen, p. 227234, et G esammelte Werke, t. 8, p. 315-322). 
D ans cette approche inédite, G adamer n'est pas du tout porté par le souci d'enrichir l'esthétique contemporaine en offrant aux visiteurs des musées ou des galeries une nouvelle possibilité d'élargir la gamme de leurs émotions au contact de la peinture moderne. Son attention demeure au contraire toute portée vers le mode d'être du tableau luimême et le rapport au monde du tableau qui s'y réalise. C'est de là que son silence peut devenir une question pour nous. $M$ algré son caractère inédit, cette question du « silence des tableaux » n'a pas eu beaucoup d'écho dans les travaux qui se sont intéressés à la critique gadamérienne de l'esthétique.

L'exploration de lieux nouveaux permet pourtant d'habiter d'une nouvellefaçon ceux qui nous paraissent plus familiers. II y a beaucoup de lieux communs dans la réflexion sur le silence et on s'y réfugie volontiers. Un séjour du côté de la peinture, fut-il bref, peut nous permettre d'écouter ensuite autrement les voix auxquelles nous croyions nous être habitués. François-M arc Gagnon invite ainsi à explorer à nouveau la contrée visitée naguère par Gadamer. L'attention demeure sans doute ici plus près des oeuvres elles-mêmes mais on notera comment ici aussi le « ductus » de la question conduit à nouveau, par-delà les limites de l'expérience « esthétique », à la question du « monde » de l'oeuvre, de celui qui advient en elle et auquel elle appartient.

Le dialogue est généralement compris comme le lieu par excellence de la parole. S'il n'est pas hostile au silence, c'est pourtant à la parole qu'il doit d'exister. En certaines circonstances exceptionnelles, on peut comprendre qu'il se réalise « aux frontières du silence ». II est beaucoup plus inhabituel qu'il soit proprement un « dialogue de silence ». O r c'est précisément ce dont il est question dans la contribution de Fabrice Blée. II s'agit de l'expérience inédite que mènent depuis plus d'une vingtaine d'années, un certain nombre de communautés monastiques bénédictines d'Europe et d'A mérique avec des communautés monastiques bouddhistes. L'importante thèse que vient de consacrer Fabrice Blée à ce sujet a montré la portée exceptionnelle de cette rencontre inédite dans le contex te actuel du dialogue interreligieux et la signification de son enracinement dans la tradition monastique multiforme des grandes traditions religieuses.

Expérience étonnante, à la vérité, surtout au moment où la question du dialogue interreligieux devient une exigence incontournable et qu'on commenceà s'énerver un peu en certains milieux devant les déplacements 
qu'elle commande. C'est avec beaucoup de doigté et avec une claire conscience de sa portée religieuse et théologique que Fabrice Blée nous introduit dans cette expérience actuelle. «Dialogue de silence», en raison, certes, deson enracinement monastique, mais aussi parce que ce dialogue prend corps « dans l'intimité de la démarche spirituelle », en deçà des débats théologiques et, comme on dit, des « échanges d'idées ». Un dialogue dans le silence où c'est le silence lui-même qui est offert en partage et qui rassemble de ce fait ceux qui le cherchent.

La contribution qui clôt ce cahier attire l'attention sur une «pratique du silence » qui nous est somme toute mal connue. Les voix de la tradition que nous appelons orthodoxe le dispute à d'autres voix étrangères et ne nous atteignent le plus souvent que comme un nouvel objet esthétique sur le marché de la consommation religieuse. $M$ élodies envoûtantes ou rituels fascinants, les figures de l'orthodoxie ne semblent pas nous parler d'abord de spiritualité exigeante ou de conversion intérieure. La « méditation silencieuse » constitue pourtant une source permanente de la fibre religieuse de l'orthodoxie. H oria R oscanu nous rappelle qu'au coeur de cette tradition niche une réalité plus discrète mais dont la puissance habite la proximité du mystère.

II ne peut y avoir à proprement parler de conclusion à un cahier comme celui-ci. Les figures du silence qui y sont évoquées sont multiples et variées. Il est bon qu'il en soit ainsi. Vouloir les ramener à tout prix à une visée commune - sous prétexte qu'il s'agirait toujours du silence - ou en dégager quelques leçons pour une tâche ou l'autre, pourrait facilement conduire à faire taire ce silence, alors qu'il s'agirait peut-être plutôt de le "garder », au même sens où le Jésus des évangiles disait qu'il fallait « écouter la Parole et la garder ». 